\title{
Healing at Home: Developing a Model for Ambulatory Alcohol "Detox" in an Aboriginal Community Controlled Health Service
}

\begin{abstract}
Introduction: Indigenous Peoples who have been colonized face a higher burden of harm from alcohol and increased risk of alcohol use disorders. Yet they often also have limited access to alcohol treatment. Limited access to withdrawal management ("detox" in particular) can be a barrier to recovery. Ambulatory or "outpatient" alcohol detox can offer improved treatment access, but no research has examined its feasibility and acceptability in Indigenous populations. Aim: To develop a model for outpatient detox delivered by an Australian Aboriginal community controlled health service (ACCHS), and to describe its feasibility and acceptability. Methods: This report describes a framework for alcohol treatment service development within an ACCHS through community, staff, and client consultation. Thematic analysis of focus groups and phone interviews were used to gain insight into the views and experiences of Aboriginal community stakeholders, service clients, and staff regarding alcohol detox services and the pilot of the outpatient detox program service model. Results: Individual, family, and community support was regarded as key to recovery from alcohol dependence. Outpatient detox was seen as a way of keeping the individual near this support. Reported positive aspects include satisfaction with the approach to care that was considered accessible, holistic, and integrated. Challenges and suggested improvements were identified. Discussion: Outpatient detox within an ACCHS was assessed as feasible and acceptable for carefully selected clients and was reported to aid access to treatment. More clinical experience is needed to fully delineate effectiveness and safety.
\end{abstract}

\section{Keywords}

Aboriginal, Australians, alcohol, withdrawal, outpatient, home, detox

\section{Authors}

Jonathan Brett, staff specialist, addiction medicine and toxicology, Drug Health Services, Sydney Local Health District; clinical lecturer, University of Sydney. Phone +61 487465199, Fax +61 95155779, Email Jonathan.Brett@sydney.edu.au.

Angela Dawson, associate professor, Australian Centre for Public and Population Health Research, Faculty of Health, University of Technology Sydney.

Rowena Ivers, general practitioner, Illawarra Aboriginal Medical Centre, Wollongong; associate professor, University of Wollongong.

Leanne Lawrence, coordinator, Drug and Alcohol Program, Illawarra Aboriginal Medical Centre, Wollongong. 
Healing at Home: Developing a Model for Ambulatory Alcohol 'Detox' in an Aboriginal Community Controlled Health Service • Jonathan Brett, Angela Dawson, Rowena Ivers, Leanne Lawrence, Sarah Barclay, Katherine Conigrave • DOI:18357/ijih121201716906

Sarah Barclay, A-Clinic coordinator, Illawarra Aboriginal Medical Centre, Wollongong.

Katherine Conigrave, senior staff specialist, addiction medicine. Drug Health Services, Sydney Local Health District; professor, Sydney Medical School, University of Sydney.

\section{Acknowledgements}

We are grateful to the community members and staff who gave their time to take part in this study. Thanks also go to Pam Simon for focus group facilitation, Christopher Ganora for conducting interviews, and Emma Brandish for research assistance. Funding to support program delivery and evaluation was provided by the Foundation for Alcohol Research and Education (FARE), with evaluation also supported by the National Health and Medical Research Council (APP1105339).

\section{Introduction}

Indigenous Peoples who have been colonized typically face a greater burden of injury, disease, and social disruption associated with alcohol use (Kirmayer, Brass, \& Tait, 2000). However, they often also encounter many barriers to accessing treatment for alcohol use disorders (Gray, Stearne, Wilson, \& Doyle, 2010). Aboriginal and Torres Strait Islander Australians (here described as Aboriginal Australians) experience 3-8 times the prevalence of alcohol-related illness, injury, and death than the general population (Calabria, Doran, Vos, Shakeshaft, \& Hall, 2010). But their barriers to treatment access for alcohol dependence include transport difficulties, fear of discrimination, and lack of culturally secure services (Brett et al., 2016; Conigrave et al., 2012; Gray, Stearne, et al., 2010; Teasdale et al., 2008).

Ensuring prompt access to quality, evidence-based, and appropriate health care for Aboriginal people with alcohol use disorders is a priority (Gray, Saggers, Wilkes, Allsop, \& Ober, 2010). This requires Aboriginal expertise in service development, delivery, and evaluation (Lee et al., 2014).

Alcohol withdrawal management ("detox") can be an essential step on the path to recovery from alcohol dependence. Untreated severe alcohol withdrawal can result in seizures and delirium tremens, while in untreated moderate alcohol withdrawal the experience of insomnia, anxiety, and intense craving can lead to a return to drinking (Saunders et al., 2016). Furthermore, those drinkers who need residential rehabilitation ("rehab") are typically asked to complete alcohol withdrawal before they can enter a rehab unit.

Medical management of alcohol withdrawal typically involves supplying a reducing dose of a long-acting benzodiazepine (typically diazepam) under health professional supervision. Thiamine (and sometimes other vitamins) is also supplied, along with psychological support and recovery planning. The diazepam treatment reduces the risk of serious complications such as seizures or delirium tremens and increases the likelihood of successful completion of withdrawal. 
Healing at Home: Developing a Model for Ambulatory Alcohol 'Detox' in an Aboriginal Community Controlled Health Service • Jonathan Brett, Angela Dawson, Rowena Ivers, Leanne Lawrence, Sarah Barclay, Katherine Conigrave • DOI:18357/ijih121201716906

In Australia, most rehabs do not have detox services on site, and the difficulty of finding a bed first in a detox and then in a rehab can be both daunting and time consuming. Residential or inpatient alcohol detox settings have limited space and often long waiting lists (Gray, Stearne, et al., 2010). This often results in missed opportunities for motivated individuals to engage with treatment.

In general populations, outpatient detox can be safe and cost-effective for carefully selected individuals (Haber, Lintzeris, Proude, \& Lopatko, 2009). Accordingly, outpatient detox is part of the Australian alcohol treatment guidelines (Cusack \& de Crespigny, 2007; Haber et al., 2009). Models have typically included daily clinic or (occasionally) home visits (Blondell, 2005; Brett, Lawrence, Ivers, \& Conigrave, 2014; Carlebach, Wake, \& Hamilton, 2011). However, there is limited understanding of the Aboriginal community's preferences for detox (Conigrave et al., 2012), and there are no studies detailing how a culturally appropriate model of care could be established and evaluated.

Anecdotally, a small number of Aboriginal community controlled health services (ACCHSs) have provided outpatient detox on an ad hoc basis (Brett et al., 2014). ACCHSs can reduce barriers to health care and improve health outcomes for Aboriginal people (Panaretto, Wenitong, Button, \& Ring, 2014). Establishing a model of outpatient detox within an ACCHS could potentially offer accessible, culturally secure, and community-prioritized health care (Gray, Stearne, et al., 2010).

Illawarra Aboriginal Medical Service (IAMS), an ACCHS in a regional town of New South Wales (NSW), Australia, had experienced difficulty accessing residential detox for clients with alcohol dependence and set out to develop and evaluate a model for outpatient detox. The objectives of this program were (a) to improve access to treatment for alcohol dependence, (b) to improve client engagement, (c) to medically manage alcohol withdrawal in a culturally safe environment, and (d) to facilitate ongoing recovery.

To develop this program, staff of the ACCHS (LL, RI) established partnerships with researchers and addiction medicine specialists $(\mathrm{AD}, \mathrm{JB}, \mathrm{KC})$ in a nearby urban centre. These academics sourced funding for program development and evaluation through a not-for-profit organization, the Foundation for Alcohol Research and Education. The authors first reviewed mainstream literature, then consulted with other services which had sporadically provided outpatient detox to Aboriginal Australians (Brett et al., 2014).

The current report describes the results of qualitative research that informed program development, the model of outpatient detox that was adopted, and the early evaluation of program feasibility and acceptability.

\section{Methods}

A descriptive qualitative study was undertaken to explore perceptions and experiences of alcohol detox during the planning and early implementation of a pilot model of outpatient home detox in IAMS, an ACCHS in regional NSW, Australia. We aimed to understand community 
Healing at Home: Developing a Model for Ambulatory Alcohol 'Detox' in an Aboriginal Community Controlled Health Service • Jonathan Brett, Angela Dawson, Rowena Ivers, Leanne Lawrence, Sarah Barclay, Katherine Conigrave • DOI:18357/ijih121201716906

knowledge and shared meaning regarding approaches to alcohol detox, as well as early client and staff experiences of the program.

We conducted interviews and focus group discussions with community members, interviews with early clients of the program, and consultations with clinic staff between October 2013 and October 2014.

The knowledge and perceptions of alcohol detox held by Aboriginal community members and ACCHS staff were explored. This qualitative information was used together with data from the previous literature review and health service consultation (Brett et al., 2014) to design a model of outpatient detox.

After the pilot program was implemented, early feedback on feasibility and acceptability was collected from clients and service providers. This was done via a client satisfaction survey, phone interviews of clients, and face-to-face interviews of service staff.

Ethical approval for this study was obtained from the Sydney Local Health District Human Research Ethics Committee and the Ethics Committee of the Aboriginal Health and Medical Research Council, NSW.

\section{Interviews and Focus Groups}

Participant selection and recruitment. Purposive criterion selection was employed to investigate in-depth cases (community leaders, clients, and staff of the project) to identify the variety of contextual and service factors that make up effective alcohol detox in this one Aboriginal community. In designing the program, local Aboriginal community stakeholders were identified by ACCHS staff and invited to participate in a focus group at the ACCHS. Additional in-depth personal perspectives were gained through one-on-one telephone interviews.

After implementation of the pilot program, the first eight clients who commenced treatment with the program were approached to participate in phone interviews. Phone interviews seemed to be the mode preferable to participants and were thought likely to optimise participation. On conclusion of their detox each client was asked by staff to complete an anonymous satisfaction survey.

Investigators also invited key staff working within the program to participate in a group consultation and face-to-face individual interviews.

Data collection. An experienced independent Aboriginal facilitator led the community stakeholder focus group. An independent non-Aboriginal interviewer (Christopher Ganora, CG) also subsequently conducted individual telephone interviews with the same participants to allow participants further opportunity to express individual opinions and clarify certain points. The same interviewer (CG) also conducted client interviews. The investigators (JB, KC) conducted group and also private individual staff consultations.

The focus group and interviews were loosely structured. Questions were formed in consultation with Aboriginal clinic staff. Areas explored included understandings of alcohol detox; experience with and views on outpatient detox; and issues associated with access to such a 
Healing at Home: Developing a Model for Ambulatory Alcohol 'Detox' in an Aboriginal Community Controlled Health Service • Jonathan Brett, Angela Dawson, Rowena Ivers, Leanne Lawrence, Sarah Barclay, Katherine Conigrave • DOI:18357/ijih121201716906

service, including acceptability and cultural appropriateness, and how access barriers could be reduced. If participants had limited knowledge of the subject, prompts were given to help stimulate recall of relevant personal experience or knowledge.

Client interviews included questions to explore pre-existing knowledge about detox, their perception of accessibility of the program, adequacy and appropriateness of information provided, satisfaction with the program, integration of aftercare, and suggestions for improvement. Staff consultation sought to determine satisfaction with program processes and outcomes.

The client satisfaction survey used a Likert scale and was completed by pen and paper.

Data analysis. The focus group and telephone interviews were recorded and transcribed verbatim. Two researchers (JB, AD) thematically analyzed the transcripts. They familiarized themselves with the transcripts and coded them independently. JB and AD met several times to discuss emergent categories and agree upon themes. Written notes were taken during and immediately after staff consultation, then typed and thematically analysed by two researchers (JB, KC). ACCHS staff and the facilitator/interviewer checked themes to ensure findings were factually correct, culturally safe, and respectful.

\section{Results}

Eight stakeholders (identified as SH1 to 8), comprising four local community Elders and four Aboriginal community members employed in government services, consented to participate in the study prior to program development.

After the pilot program had been developed and implemented, four of the eight clients who completed the program (CL1 to 4) consented to interviews, while four declined. Due to the small size of the community, further participant characteristics are not revealed to preserve anonymity. Seven program staff participated in consultations: one general practitioner (GP), one GP trainee, two practice nurses, and three Aboriginal drug and alcohol workers.

The themes that emerged from interviews and focus groups are described below.

\section{Interviews and Focus Groups to Inform Model of Service Delivery}

Lived experience of nature and effects of alcohol misuse. All community stakeholder participants had personal or professional experience of alcohol misuse and related consequences, and all acknowledged that alcohol was a problem within the local Aboriginal community. The intergenerational nature of alcohol misuse, trauma, and disempowerment was described.

Resilience. Despite the difficulties of their personal experiences, participants expressed their ability to adapt to stress and adversity. Resilience was expressed through participant descriptions of needing to remain strong and to keep the family together. There was generally a feeling of hope for the future, and all stakeholders expressed the need to be proactive in dealing 
Healing at Home: Developing a Model for Ambulatory Alcohol 'Detox' in an Aboriginal Community Controlled Health Service • Jonathan Brett, Angela Dawson, Rowena Ivers, Leanne Lawrence, Sarah Barclay, Katherine Conigrave • DOI:18357/ijih121201716906

with alcohol problems: "Shake your eyebrows and let all that dust, all that grit fall off you. Then you can see a beauty that surrounds you when you don't have alcohol” (SH8).

Understanding of detox and service needs. There was a general understanding that withdrawing from alcohol could be difficult: "When detoxing it can become a rough thing" (SH3). A few of the community stakeholders had personal experience of alcohol withdrawal. However, only one identified the need for medical withdrawal management. "They can't go cold turkey with it straight away because it might have other health effects" (SH1).

Several participants described alcohol withdrawal in the literal sense of "detoxification," a feeling that the body was contaminated with toxins that needed to be purged. "If you can get in there early enough you can clean them out ... it means cleaning your body of all those dreadful things that come from alcohol" (SH2). A minority of community stakeholders understood the process of medical management of alcohol withdrawal as involving medication with benzodiazepines and regular monitoring.

One participant identified the lack of options for detox as a barrier to entering rehabilitation. However, there was a general confusion between detox and rehab, with most participants thinking that the two were synonymous.

The theme of the drinker needing to be isolated in order to treat alcohol "problems" emerged during several interviews: "They locked them away until they come home, fully clean of alcohol" (SH7).

All stakeholders identified the need for more and improved local alcohol treatment services. This included a need for services to deal with complex needs, such as psychiatric comorbidity.

Motivation. Engagement and remaining motivated were considered central issues in treatment. Motivation was perceived at the level of the individual, the family, and the community. Furthermore, recovery was often expressed as a journey: "That's a journey the family can take together" (SH1).

Views on acceptability; defining the model. Participants expressed a range of views on what they felt would constitute an acceptable outpatient alcohol detox service (Table 1):

Cultural safety. Trust was seen as a major factor for most people in terms of who provided the service: "Let them put their trust in you first" (SH6). There was a feeling that with the right services, support, and commitment, outpatient detox was acceptable. "It's culturally comfortable for them to be there if they have the right support through services who may assist them on a daily or weekly basis to commit to their journey" (SH3).

Shame and privacy. Shame was seen as a barrier to help seeking, in particular in group treatment environments. What is more, group settings could lead to leakage of personal information to the wider Aboriginal community. "If someone wants to detox, they can do it from 
Healing at Home: Developing a Model for Ambulatory Alcohol 'Detox' in an Aboriginal Community Controlled Health Service • Jonathan Brett, Angela Dawson, Rowena Ivers, Leanne Lawrence, Sarah Barclay, Katherine Conigrave • DOI:18357/ijih121201716906

home. I'm sure that a lot of people would put their hand up rather than ... to have to face their demons and be brought out, like shunned or put up on the limelight in the community and say that this person has an issue around alcohol" (SH3).

Issues with mainstream services/preference for Aboriginal controlled services. Both community stakeholders and clients felt that some Aboriginal people would prefer an Aboriginal specific service: "I know a lot wouldn't go to the mainstream one, they would only go to the Indigenous one" (SH4). Some of them also related negative personal experiences within mainstream services: "They just don't like the hospital thing because they think ... nurses and everyone doing wrong by them" (SH7). Therefore, providing outpatient detox through an ACCHS was generally seen as preferable.

Keeping the family together. Being able to keep the family together through treatment, to understand the family context, and even to engage the family during detox and beyond was an emergent theme. This also related to family commitment as an important motivator. It was appreciated that these family-focused goals could be achieved with an outpatient withdrawal program. "They want to be close to their families for support, so they can talk to them and be there for them. It's just too difficult when they know they've got to travel a couple of hundred kilometres away. They just give up" (SH1).

Focusing on community for recovery. Reconnecting with community to share experiences and information was seen as an important motivator and identified as a priority to integrate into the program. Stakeholders talked about the importance of accessing men's and women's groups through the program as a way to achieve this and to prevent alcohol harms in others.

The need for individuals within the community to serve as champions to promote new services such as this program was described: "They've got to get the right information to the right people that want to do the detox" (SH1).

A community need for services such as the program to engage and assist Aboriginal youth drinkers was also expressed: "Get through to the kids and say 'Do you want to end up like that?"” (SH2).

Accessible and streamlined. One of the issues expressed by ACCHS staff prior to the opening of the program was that existing detox services were difficult to access because of the number of assessments clients have to go through. These assessments could occur over several days, resulting in a high client attrition rate.

Transport was seen as a major barrier to accessing care at other mainstream services: "Transport is another difficulty ... a lot of them don't have cars" (SH4).

Commitment and planning for the future. Commitment of the program staff to the clients was seen as essential. "If you're going to make it a home detox, you just need to keep ... 
Healing at Home: Developing a Model for Ambulatory Alcohol 'Detox' in an Aboriginal Community Controlled Health Service • Jonathan Brett, Angela Dawson, Rowena Ivers, Leanne Lawrence, Sarah Barclay, Katherine Conigrave • DOI:18357/ijih121201716906

on top of it, a constant check-in to keep them motivated and focused on what they're achieving" (SH3).

All participants felt that the journey did not end following detox and that ongoing commitment from all involved was important: “It doesn't just end when they're in there; they've got to have that follow-on when they leave" (SH6).

The need for relapse prevention approaches even during the detox process was described by community stakeholders.

Peer support and champions for the program. The importance of positive early community experience of the program for its ongoing success was voiced. The concepts of peer support and role models were raised, in which individuals who had successfully completed the program could promote it or support their peers through the process: "You just need one or two people to give the program [a try] for it to be successful and they'll probably carry the flag for you" (SH1).

\section{Table 1}

Summary of the Characteristics Desired in an Outpatient Detox and the Challenges of Outpatient Detox According to Community Stakeholders

\begin{tabular}{|c|c|}
\hline Desired Characteristics & Challenges \\
\hline - Cultural safety & - Other drinkers may be nearby \\
\hline - Privacy / avoid shame & $\begin{array}{l}\text { - Boredom and all the usual triggers to } \\
\text { relapse may be present }\end{array}$ \\
\hline $\begin{array}{l}\text { - Preference for Aboriginal controlled } \\
\text { services }\end{array}$ & $\begin{array}{l}\text { A new service will take time to } \\
\text { engender trust }\end{array}$ \\
\hline - Keep family together & \\
\hline - Focusing on community for recovery & \\
\hline - Accessible and streamlined & \\
\hline $\begin{array}{l}\text { - Commitment to clients and planning } \\
\text { for the future }\end{array}$ & \\
\hline $\begin{array}{l}\text { - Peer support and champions for the } \\
\text { program }\end{array}$ & \\
\hline
\end{tabular}

\section{Anticipated Challenges}

Participants anticipated several challenges to the outpatient withdrawal program (Table 1). Having other drinkers in the home environment was a common theme: "If someone else walks into the house with a bottle in their hands, that's when they could fall back" (SH4). Some of the participants expressed a concern that drinkers could be exposed to all of the triggers to drink in their usual environment: "It's a comfortable environment for them. It's so easy for them to just slip back into their habits" (SH3). Several community stakeholders felt that if people are 
Healing at Home: Developing a Model for Ambulatory Alcohol 'Detox' in an Aboriginal Community Controlled Health Service • Jonathan Brett, Angela Dawson, Rowena Ivers, Leanne Lawrence, Sarah Barclay, Katherine Conigrave • DOI:18357/ijih121201716906

isolated in their own homes, then boredom and difficulty coping with cravings due to the lack of distractions may lead to relapse.

One participant felt that people may take some time to trust and engage with a new service: "A lot of black fellas, they're always unsure of the unknown" (SH1).

\section{The Development of a Model for Outpatient Detox}

Existing scientific literature (Blondell, 2005; Carlebach et al., 2011; Gray, Saggers, et al., 2010; Hayashida et al., 1989; Sannibale, Fucito, O’Connor, \& Curry, 2005), in conjunction with findings from the analysis of the focus groups and interviews, and consultation with other services (Brett et al., 2014), ACCHS staff, and the ACCHS board, informed the design of the pilot outpatient detox program that was implemented in the alcohol and other drugs section of the ACCHS.

Withdrawal management was always initiated on a Monday or Tuesday to reduce the risk of complications occurring over the weekend. Clients were offered transport to the clinic each morning and the option of a home visit or phone call in the afternoon. The standard detox program lasted for 5 days and involved a daily dispensed, weaning regime of diazepam, along with thiamine, supportive counselling, and relapse-prevention planning. The following week, clients received medical follow-up, and counselling or other follow-up as appropriate. They received follow-up phone calls from clinic staff at 2 and 6 weeks.

The ACCHS relied on usual staff of the ACCHS, plus an additional half-time alcohol and other drugs counsellor. Staff identified individuals who could potentially benefit from alcohol detox from within the ACCHS patient database and sent them letters advertising the new service. Promotional and educational activities were also undertaken within the ACCHS and through local media.

Clients could be referred for initial assessment for the program if a staff member suspected they had an alcohol use disorder. Clients could also self-refer. Attempts were made to provide same-day assessments by the clinic coordinator and a general practitioner to determine suitability for outpatient detox using national guidelines (Haber et al., 2009). In brief, patients were eligible if they were considered to be at risk of alcohol withdrawal, had no past history of severe withdrawal (including seizures or delirium tremens), no other active substance use (other than cannabis), no unstable medical or psychiatric illness, and stable accommodation with no drinkers at home. The presence of a support person was also preferable.

If considered suitable, the client was informed about the program. A relapse prevention plan was formed before initiating treatment. For those considered not suitable for outpatient detox, referral to residential services was offered. The program had the capacity to accept one to two clients per week.

\section{Experience of Clients and Staff in the Pilot Phase of the Program}

Clients and staff identified a number of advantages to outpatient detox delivered through an ACCHS, but challenges and suggestions for improvement were also identified. 
Healing at Home: Developing a Model for Ambulatory Alcohol 'Detox' in an Aboriginal Community Controlled Health Service • Jonathan Brett, Angela Dawson, Rowena Ivers, Leanne Lawrence, Sarah Barclay, Katherine Conigrave • DOI:18357/ijih121201716906

Challenges and advantages of an outpatient or home setting for detox. As anticipated by stakeholder consultation, the home setting meant potential isolation and lack of 24-hour support for the drinker: "They came out to your house to see you, but I had a lot of time for thinking ... with boredom you end up thinking, thinking, thinking and back on the grog" (CL4).

The same client valued being able to develop life skills and self-efficacy on his own terms and own environment: "If you don't get out and learn and things — skills of mingling again-it won't happen" (CL4).

Accessible and streamlined care. The program was designed to streamline the treatment process. One client expressed his satisfaction with this aspect: "So I had one appointment in the morning and at lunchtime I was at [the ACCHS] and I was on the program" (CL2).

Satisfaction was expressed with the provision of transport to and from the program.

Time spent talking in the car while being transported to and from the clinic was also seen as important by both clients and staff to engage clients in relapse prevention in a non-threatening environment.

Integrated and ongoing care. All eight clients completing the program remained engaged after treatment at 6-week follow-up, with five remaining abstinent until that time. This included accessing counselling, taking relapse prevention medications, or, in one case, transitioning to residential rehabilitation immediately after detox.

Holistic care. While engaged with the program, clients described how other health and social needs were addressed. Two of the clients described that their mental health problems were identified and treated. "They gave me other medications for ... because I was really, really depressed because I hadn't seen my daughter for at least six and a half months" (CL2).

One client described how he was able to access hearing aids, ophthalmology services, and a dietician.

All clients were registered with the "Close the Gap" scheme (Australian Government Department of Human Services, 2016), which provided the diazepam or other medicines at subsidized rates. Two of the clients were also able to register for a state debt repayment scheme in which their treatment involvement could be used to reduce state debt (NSW Office of State Revenue, 2016), and one was able to register for a parenting course.

A period of sobriety allowed two of the clients to engage with men's groups, one of which elected the client to its board of directors.

Commitment to community and optimism. One client expressed the importance of both community support and giving back to the community: "Doing regular with that [the men's group] - that's helping me communicate with kids and get it into their head don't drink, look what I've gone through" (CL4). A sense of optimism was conveyed: "At the end of the tunnel there is a light; there is goals to be achieved" (CL3). 
Healing at Home: Developing a Model for Ambulatory Alcohol 'Detox' in an Aboriginal Community Controlled Health Service • Jonathan Brett, Angela Dawson, Rowena Ivers, Leanne Lawrence, Sarah Barclay, Katherine Conigrave • DOI:18357/ijih121201716906

Interactions with staff. Clients were all satisfied with the verbal and written information they were given. They felt staff were compassionate, approachable, and honest: "I didn't feel intimidated or inadequate at all, didn't feel pressured or embarrassed" (CL2). They also valued home visits. Often this was seen as a break from boredom and isolation.

Staff perceptions. Staff reported no major adverse events during the pilot phase of the program, and in particular no seizures or overdoses from the combination of alcohol and benzodiazepines.

Staff generally felt positive about the pilot program; however, they felt that clearly defining roles and responsibilities with early managerial support was important. Some medical staff felt that for selected clients, a less intensive model of care could be adopted with fewer clinic visits.

Diazepam dispensing processes were perceived to be a challenge, particularly as regulations required dispensing to be done in the presence of a medical officer or by a pharmacist. Near the end of the pilot program efforts were made to streamline the dispensing process by forming relationships with a local pharmacist, who could provide the medicines in advance in a Webster-pak (Elliott, 2014). This pack could then be cut into strips (appropriately labelled), and nurses could hand one strip to the client daily. Other clinic processes were streamlined as the evaluation was conducted, including reducing or deferring non-core assessments or paperwork until after withdrawal.

Identifying clients who could be suitable for the clinic was also a challenge. Staff felt there were many clients within the ACCHS who had an alcohol use disorder but had not yet been identified or offered assessment.

Staff expressed satisfaction with improved links that had been built with local mainstream residential and hospital services as part of the program development. This allowed rapid access to mainstream withdrawal and rehabilitation services when needed.

Quantitative feedback from satisfaction surveys of the first clients. Eight clients had 12 episodes of care during the pilot phase of the program (two had two episodes and one had three). After each episode, clients were able to complete an anonymous satisfaction survey. Ten surveys were received. All surveys indicated that clients were satisfied or very satisfied with the program. They all indicated they felt welcome in the clinic and were happy with staff providing the service and with information they had received. All would consider repeating the program if necessary and all would recommend it to a friend. All clients reported they were offered followup. Clients stated in feedback forms that they appreciated the chance to have a break from drinking and the help they received.

\section{Discussion}

Study participants expressed a pressing need for improved access to alcohol detox services for Aboriginal Australians. This is consistent with previous consultations with the 
Healing at Home: Developing a Model for Ambulatory Alcohol 'Detox' in an Aboriginal Community Controlled Health Service • Jonathan Brett, Angela Dawson, Rowena Ivers, Leanne Lawrence, Sarah Barclay, Katherine Conigrave • DOI:18357/ijih121201716906

Aboriginal community and with service providers (Gray, Stearne, et al., 2010). Various models of outpatient alcohol detox for general populations have been shown to be safe and effective (Alwyn, John, Hodgson, \& Phillips, 2004; Blondell, 2005; Carlebach et al., 2011; Hayashida et al., 1989). This study involved a collaborative approach to developing a model of outpatient detox in an Aboriginal community, drawing on the unique opportunities for trust and accessibility provided by a community controlled health service.

Our study found a lack of community understanding of the medical management of alcohol withdrawal. Community discourse revealed the stigma associated with alcohol problems and treatment that involved being separated from community and locked away. These perceptions may echo similar traumas experienced within Aboriginal communities and are likely to pose an additional barrier to treatment access. A lack of understanding of the broad range of alcohol treatment options has been described in other contexts (Brady, Dawe, \& Richmond, 1998). There is a need for community education on the full range of available alcohol treatments as well as on the role of outpatient detox specifically. Ideally Aboriginal champions within communities should provide this education to encourage open dialogue regarding alcohol issues affecting the community and encourage access to local treatment services.

The importance of services being sensitive to potential desire for involvement of family and community in treatment was also highlighted. This is consistent with previous studies of Aboriginal health (Teasdale et al., 2008). The model of outpatient withdrawal employed in this study appears to facilitate this involvement.

Concerns about other drinkers in the house did not seem to affect clients commencing the program. This may reflect careful screening of clients and their environment for suitability by staff. In the pilot phase of the program eight clients commenced outpatient detox. Five of these remained abstinent at 6-week follow up. Of these clients, all had been offered alcohol relapse prevention medication and were receiving psychosocial support either via counselling or support groups. Three clients relapsed to heavy drinking either during or shortly after the program (which was the reason for non-completion in all instances) and had further attempts at outpatient detox but eventually required residential treatment.

It was noted that despite the perceived magnitude of the drinking problem within the community, the rate of initial service use was low. Staff felt that enhanced screening and detection of unhealthy drinking within the primary health section of the ACCHS would help to identify clients with an earlier stage of dependence, a lower risk of complicated withdrawal, and therefore greater suitability for outpatient detox.

When establishing an outpatient withdrawal clinic it is important that staff feel supported by senior management, have adequate resources and training, and play clearly defined roles. There is also need for ongoing program evaluation and feedback to staff, clients, and community to assist in continual refinement of the model. The pilot program gave high priority to client contact, with twice daily face-to-face patient contact in most cases in order to minimize the chance of any adverse client event and maximize client support. This made the program relatively labour intensive. Where resources are limited, a less resource intensive approach may 
Healing at Home: Developing a Model for Ambulatory Alcohol 'Detox' in an Aboriginal Community Controlled Health Service • Jonathan Brett, Angela Dawson, Rowena Ivers, Leanne Lawrence, Sarah Barclay, Katherine Conigrave • DOI:18357/ijih121201716906

be appropriate for selected clients. This may include pharmacy rather than clinic-based collection of medicines and omitting the afternoon home visit in cases where it is not deemed necessary. However, this model has not yet been evaluated in an Aboriginal context, and risks need to be carefully assessed.

While service changes require consultation and time, the process of developing a program such as this was observed to yield wider benefits such as staff skills development and improved relationships with mainstream services.

\section{Limitations}

This study has described the process of program development and early pilot evaluation. A larger study is required to more clearly delineate safety and effectiveness of this model of care; however, international evidence suggests that in carefully selected clients outpatient detox is a valid treatment choice. This study was conducted in a regional centre, and care must be taken in extrapolating these findings to rural and remote locations in which staffing and service availability may be limited and client health needs may be even more complex. However, the process of model development outlined here could be employed to develop locally appropriate models of care for outpatient alcohol detox.

\section{Conclusion}

This study demonstrated the potential for an Australian Aboriginal community controlled health service to provide outpatient alcohol detox treatment that is accessible and acceptable. Similar approaches to developing and evaluating a model of care for alcohol withdrawal may be valuable within other settings in Australia and in other Indigenous or marginalized populations worldwide.

\section{References}

Alwyn, T., John, B., Hodgson, R. J., \& Phillips, C. J. (2004). The addition of a psychological intervention to a home detoxification programme. Alcohol and Alcoholism, 39(6), 536541. doi:10.1093/alcalc/agh092

Australian Government Department of Human Services. (2016). Closing the gap-PBS copayment measure [Web page]. Retrieved from https://www.humanservices.gov.au/health-professionals/services/medicare/closing-gappbs-co-payment-measure

Blondell, R. D. (2005). Ambulatory detoxification of patients with alcohol dependence. American Family Physician, 71(3), 495-502.

Brady, M., Dawe, S., \& Richmond, R. (1998). Expanding knowledge among Aboriginal service providers on treatment options for excessive alcohol use. Drug and Alcohol Review, 17(1), 69-76. doi:10.1080/09595239800187611 
Healing at Home: Developing a Model for Ambulatory Alcohol 'Detox' in an Aboriginal Community Controlled Health Service • Jonathan Brett, Angela Dawson, Rowena Ivers, Leanne Lawrence, Sarah Barclay, Katherine Conigrave • DOI:18357/ijih121201716906

Brett, J., Lawrence, L., Ivers, R., \& Conigrave, K. (2014). Outpatient alcohol withdrawal management for Aboriginal and Torres Strait Islander peoples. Australian Family Physician, 43(8), 563-566.

Brett, J., Lee, K. S. K., Gray, D., Wilson, S., Freeburn, B., Harrison, K., \& Conigrave, K. (2016). Mind the gap: What is the difference between alcohol treatment need and access for Aboriginal and Torres Strait Islander Australians? Drug and Alcohol Review, 35(4), 456460. doi:10.1111/dar.12313

Calabria, B., Doran, C. M., Vos, T., Shakeshaft, A. P., \& Hall, W. (2010). Epidemiology of alcohol-related burden of disease among Indigenous Australians. Australian and New Zealand Journal of Public Health, 34(s1), S47-S51. doi:10.1111/j.17536405.2010.00553.x

Carlebach, S., Wake, D., \& Hamilton, S. (2011). Experiences of home detoxification for alcohol dependency. Nursing Standard, 26(10), 41-47. doi:10.7748/ns.26.10.41.s50

Conigrave, K., Freeman, B., Caroll, T., Simpson, L., Lee, K., Wade, V., ... Freeburn, B. (2012). The Alcohol Awareness project: Community education and brief intervention in an urban Aboriginal setting. Health Promotion Journal of Australia, 23(3), 219-225.

Cusack, L., \& de Crespigny, C. (2007). Alcohol treatment guidelines for Indigenous Australians. Retrieved from University of Adelaide website: http://hdl.handle.net/2440/79677

Elliott, R. A. (2014). Appropriate use of dose administration aids. Australian Prescriber, 37(2), 46-50. doi:10.18773/austprescr.2014.020

Gray, D., Saggers, S., Wilkes, E., Allsop, S., \& Ober, C. (2010). Managing alcohol-related problems among Indigenous Australians: What the literature tells us. Australian and New Zealand Journal of Public Health, 34(s1), S34-S35. doi:10.1111/j.17536405.2010.00550.x

Gray, D., Stearne, A., Wilson, M., \& Doyle, M. (2010). Indigenous-specific alcohol and other drug interventions: Continuities, changes and areas of greatest need (ANCD Research Paper 20). Canberra, Australia: Australian National Council on Drugs.

Haber, P., Lintzeris, N., Proude, E., \& Lopatko, O. (2009). Guidelines for the treatment of alcohol problems. Retrieved from Australian Government Department of Health website: https://www.health.gov.au/internet/main/publishing.nsf/Content/0FD6C7C289CD31C9C A257BF0001F96BD/\$File/AustAlctreatguidelines 2009.pdf

Hayashida, M., Alterman, A. I., McLellan, A. T., O’Brien, C. P., Purtill, J. J., Volpicelli, J. R., ... Hall, C. P. (1989). Comparative effectiveness and costs of inpatient and outpatient detoxification of patients with mild-to-moderate alcohol withdrawal syndrome. The New England Journal of Medicine, 320, 358-365. doi:10.1056/NEJM198902093200605

Kirmayer, L. J., Brass, G. M., \& Tait, C. L. (2000). The mental health of Aboriginal Peoples: Transformations of identity and community. Canadian Journal of Psychiatry, 45(7), 607-616. doi:10.1177/070674370004500702 
Healing at Home: Developing a Model for Ambulatory Alcohol 'Detox' in an Aboriginal Community Controlled Health Service • Jonathan Brett, Angela Dawson, Rowena Ivers, Leanne Lawrence, Sarah Barclay, Katherine Conigrave • DOI:18357/ijih121201716906

Lee, K. S. K., Chikritzhs, T., Wilson, S., Wilkes, E., Gray, D., Room, R., \& Conigrave, K. M. (2014). Better methods to collect self-reported alcohol and other drug use data from Aboriginal and Torres Strait Islander Australians. Drug and Alcohol Review, 33(5), 466472. doi:10.1111/dar.12159

NSW Office of State Revenue. (2016). Work and development order [Web page]. Retrieved from http://www.sdro.nsw.gov.au/fines/eo/wdo.php?region=mnc

Panaretto, K. S., Wenitong, M., Button, S., \& Ring, I. T. (2014). Aboriginal community controlled health services: Leading the way in primary health care. The Medical Journal of Australia, 200(11), 649-652. doi:10.5694/mja13.00005

Sannibale, C., Fucito, L., O’Connor, D., \& Curry, K. (2005). Process evaluation of an out-patient detoxification service. Drug and Alcohol Review, 24(6), 475-481. doi:10.1080/09595230500292912

Saunders, J. B, Conigrave, K. M., Latt, N. C., Nutt, D. J., Marshall, E. J., Ling, W., \& Higuchi, S. (Eds.) (2016). Addiction medicine (2nd ed.). Oxford, UK: Oxford University Press.

Teasdale, K. E., Conigrave, K. M., Kiel, K. A., Freeburn, B., Long, G., \& Becker, K. (2008). Improving services for prevention and treatment of substance misuse for Aboriginal communities in a Sydney Area Health Service. Drug and Alcohol Review, 27(2), 152159. doi:10.1080/09595230701829447 